\title{
Successful laparoscopic sacro-colpopexy with a mesh for sigmoid neovaginal prolapse: Case report and Discussion
}

\author{
Xue Jiao ${ }^{1}$, Ming Yuan ${ }^{1}$, Miaomiao $\mathrm{Ji}^{1}$, and Guoyun Wang ${ }^{1}$ \\ ${ }^{1}$ Shandong University Qilu Hospital
}

November 4, 2020

\begin{abstract}
For women with congenital vaginal agenesis and sexual needs, creating a functioning neovagina is a practical therapeutic schedule. Colpopoiesis using the sigmoid intestine were more reported in the literatures. Though the incidence of neovaginal prolapse (NP) is relatively low (3-8\%), it does bring great inconvenience to patients, and expose gynecologists to tough challenges. Hereon, we report a case of NP 10 years after sigmoid colon vaginoplasty that was successfully repaired by laparoscopic sacrocolpopexy with a mesh, hoping to provide meaningful evidence for this rare situation.
\end{abstract}

Title page

Title: Successful laparoscopic sacro-colpopexy with a mesh for sigmoid neovaginal prolapse: Case report and Discussion.

Authors: Xue Jiao ${ }^{\mathrm{a}}$, Yuan Ming ${ }^{\mathrm{a}}$, Miaomiao $\mathrm{Ji}^{\mathrm{a}}$, Guoyun Wang, ${ }^{\mathrm{a}}$

Author affiliations and addresses: ${ }^{a}$ Department of Obstetrics and Gynecology, Qilu Hospital of Shandong University, Jinan, 250012, China.

Corresponding author:Guoyun Wang. E-mail:wangguoy@sdu.edu.cn. Address: NO.107 Wenhuaxi Road, Jinan, Shandong, 250012, China. Tel +86-1856 0081729.

Running Head: Laparoscopy for sigmoid neovaginal prolapse

\section{Main body of text}

\section{Introduction}

For women with congenital vaginal agenesis and sexual needs, creating a functioning neovagina is a practical therapeutic schedule, and different methods have been reported in the literature. Nowadays, non-operative vaginal dilation is the preferred treatment proposed by the American Society of Obstetrics and Gynecology ${ }^{[1]}$. Besides, tissues such as peritoneum and intestinal segments were frequently used for colpoplasty, especially the sigmoid colon. In the early 1980s, colpopoiesis using the sigmoid intestine was first reported by Goligher $^{[2]}$. The advantages of this tissue include efficient blood supply and self-lubrication, and regular vaginal dilation is not required. In a meta-analysis, Horbach reported that the overall rate was about $6.4 \%$ [3]. However, although the rate is lower than other treatment, it is also associated with various postoperative complications, including postoperative ileus, ulcerative colitis and adenocarcinoma of neovagina ${ }^{[4]}$. Though the incidence of NP is relatively low (3-8\%), it does bring great inconvenience to patients, and expose tough challenge to the gynecologists, for there is no accordant and standard repair solution presented up to now ${ }^{[5]}$. Hereon, we report a case of NP 10 years after sigmoid colon vaginoplasty that was successfully repaired by laparoscopic sacro-colpopexy with a mesh, hoping to provide meaningful evidence for this rare situation. 


\section{Methods and results}

A 32-year-old woman came to our obstetrics and gynecology clinic for evaluation and treatment of NP. She underwent transabdominal sigmoid vaginoplasty 10 years previously in another hospital. Three months later, she had satisfying sexual intercourse with her husband, and did not perform vaginal dilation. Two years ago, she found a mass protruding from vaginal introitus, aggravating when she was standing for a long time or coughing and so on increased abdominal pressure. In addition, she was troubled by the increasing foul-smelling vaginal discharge, which seriously affected her daily life and sexual intercourse. The patient requested surgical repair. Gynecologic examination showed exstrophy of the vaginal apex 4 centimeters beyond the hymen (Figure S1). Gynecological sonography prompted no uterus was detected, and no obvious abnormality was found in urinary system examination. Pelvic magnetic resonance revealed malformation of reproductive system, and vaginal wall was slightly thickened.

After well informing the patients about the risks and benefits of using a mesh and laparoscopic operations, and obtaining the informed consent of the patient, we performed the laparoscopic sacro-colpopexy with a mesh. Under the general anesthesia, the patient was placed in the lithotomy position. During the operation, we recognized intestines were compact adherent to the parietal peritoneum. After blunt and sharp dissection, we observed the absence of uterus and right adnexa in pelvic cavity. Using the uterine manipulator to explore the sigmoid neovagina, and the original bowel was located on the left of artificial vagina. For the vascular pedicle locating on the posterior wall of vagina, we could only completely separate the anterior wall. After carefully exploring the anterior region of sacral vertebra, we opened the right retroperitoneum from the sacral promontory, as well as dissected and isolated the right ureter. The mesh was sutured at the anterior wall and apex of neovagina (Figure 1), and then was suspended in the anterior sacral region without blood vessels (Figure 2). Then peritoneum was closed with interrupted suture with absorbable thread to posit the mesh completely retroperitoneal (Figure 3). After surgery, we performed the gynecological examination again, and there was no prolapse of neovagina (Figure 4).

The operation lasted 195 minutes. The postoperative course was uneventful without any mesh related complications. The patient was discharged 6 days after surgery. One month after operation, the patient returned for reexamine. No neovagina prolapse was detectable (Figure S2), and the patient presented significant improvement of symptoms and satisfied with sexual intercourse.

\section{Comments}

Prolapse of the sigmoid neovagina is scarcely reported in the literature, one study reviewed that the incidence of prolapse of postoperative sigmoid neovagina was $2.3 \%{ }^{[6]}$. However, its pathogenesis and treatment are still unclear. The pathogenesis might be related to the lack of supporting structure and the length of sigmoid neovagina ${ }^{[7]}$. Normally, the vagina is divided into two parts according to the origin. The upper two-thirds originate from the mesoderm (Mullerian's duct), but others originate from endoderm (urogenital sinus $)^{[8]}$. The upper part is anchored by the endopelvic connective tissue to the pelvic wall or sacrum, and the middle and lower part is fused to the arcus tendineus fascia pelvis and perineum. For women with congenital vaginal agenesis or Mayer-Rokitansky-Küster Hauser Syndrome, no matter which vaginoplasty was performed, it is impossible to perfect reconstruction these support structures ${ }^{[9]}$. Another related reason for the prolapse is neovaginal length. Though using sigmoid colon creating a neovagina could achieve good neovaginal depth, people after vaginoplasty also occurred NP, including mucosal prolapse and vaginal vault prolapse $^{[10]}$. Vaginal vault prolapse might be closely related to short neovaginal ${ }^{[7]}$, as well as elongation and loosening of supporting tissues, but a large number of epidemiological studies are needed to prove evidence.

The current recommended treatment for mild mucosal prolapse is surgical removal or fulgerize the redundant tissue ${ }^{[11]}$. Nevertheless, there are no guidelines for vault prolapse or severe mucosal prolapse, because few literature reported this kind of cases, and the risk of recurrence is still uncertain. Matsui reported the first case of prolapse of a sigmoid neovagina that was required with retroperitoneal sacropexy in 1999, and the results are satisfactory ${ }^{[12]}$. Rei Yokomizo reported two patients developed severe mucosal prolapse repairing by resecting the redundant sigmoid or replacement therapy by a connected skin graft, but the former has 
recurred $^{[10]}$. Some cases reported that suspending the prolapsed neovagina with transabdominal sacral colpopexy with or without paravaginal repair was an effective treatment ${ }^{[13]}$. In addition, suspending the neovagina to the sacrospinous ligament with or without a mesh has been reported in literatures and successfully repaired the prolapse ${ }^{[14]}$. In recent years, for the advantages of light surgical trauma and faster postoperative recovery, laparoscopic surgery has been successfully applied in the disposal of $\mathrm{NP}^{[15]}$. Sacrospinous ligament fixation and sacro-colpopexy turn into be valid surgical options of treatment, and using mesh repairing could enhance the support of the upper neovagina ${ }^{[16]}$. However, the long-term efficacy and safety of the mesh have not been clear. Overall, the choice of exact treatment of patients with NP requires to be combined with the anatomy, the length and width of the neovagina, as well as demands of patients for daily life and sexual intercourse. Well-designed studies and more case reports are needed to access the outcome and safety of various treatment.

In conclusion, we reported a case of NP10 years after sigmoid vaginoplasty that was successfully repaired by laparoscopic sacro-colpopexy with a mesh. After adequately preoperative evaluation and careful operation, the patient recovered well without any complications. Laparoscopic sacro-colpopexy might be an effective and safe treatment for NP. We will continue to follow up the outcome of this patient, as well as the complications of the surgery and mesh.

\section{Disclosure of interests}

There is no conflict of interest to declare.

\section{Contribution to authorship}

Data acquisition, analysis and interpretation, drafting the article and final approval of the version to be published (Xue Jiao). Data acquisition, the surgical procedure, analysis and interpretation, final approval of the version to be published (Ming Yuan, Miaomiao Ji). Responsibility for the initial concept, design of study and final review of the manuscript (Guoyun Wang).

\section{Details of patient's consent}

This study obtained the patient's permission to publication.

\section{Funding}

This study was not funded.

\section{References}

[1] Callens N, De Cuypere G, De Sutter P, Monstrey S, Weyers S, Hoebeke P, et al. An update on surgical and non-surgical treatments for vaginal hypoplasia. Hum Reprod Update. 2014;20:775-801.

[2] Goligher JC. The use of pedicled transplants of sigmoid or other parts of the intestinal tract for vaginal construction. Ann R Coll Surg Engl. 1983;65:353-5.

[3] Horbach SE, Bouman MB, Smit JM, Özer M, Buncamper ME, Mullender MG. Outcome of Vaginoplasty in Male-to-Female Transgenders: A Systematic Review of Surgical Techniques. J Sex Med. 2015;12:1499-512.

[4] Djordjevic ML, Stanojevic DS, Bizic MR. Rectosigmoid vaginoplasty: clinical experience and outcomes in 86 cases. J Sex Med. 2011;8:3487-94.

[5] Ferrando CA. Vaginoplasty Complications. Clin Plast Surg. 2018;45:361-8.

[6] Kondo W, Ribeiro R, Tsumanuma FK, Zomer MT. Laparoscopic promontofixation for the treatment of recurrent sigmoid neovaginal prolapse: case report and systematic review of the literature. J Minim Invasive Gynecol. 2012;19:176-82.

[7] Hao Z, Yang S. Neovaginal Prolapse Treated with Sacrospinous Ligament Suspension: A Case Report and Review of the Literature. J Pediatr Adolesc Gynecol. 2017;30:505-7. 
[8] Schaffer J, Fabricant C, Carr BR. Vaginal vault prolapse after nonsurgical and surgical treatment of MAAdullerian agenesis. Obstet Gynecol. 2002;99:947-9.

[9] Maher CF, Baessler KK, Barber MD, Cheong C, Consten ECJ, Cooper KG, et al. Surgical management of pelvic organ prolapse. Climacteric. 2019;22:229-35.

[10] Yokomizo R, Murakami T, Naitou H, Yamada A. Treatment for prolapse of the sigmoid neovagina in Mayer-Rokitansky-Kuster-Hauser syndrome. Obstet Gynecol. 2002;100:1085-7.

[11] Yang B, Wang N, Zhang S, Wang M. Vaginal reconstruction with sigmoid colon in patients with congenital absence of vagina and menses retention: a report of treatment experience in 22 young women. Int Urogynecol J. 2013;24:155-60.

[12] Matsui H, Seki K, Sekiya S. Prolapse of the neovagina in Mayer-Rokitansky-Kuster-Hauser syndrome. A case report. J Reprod Med. 1999;44:548-50.

[13] Calcagno M, Pastore M, Bellati F, Plotti F, Maffucci D, Boni T, et al. Early prolapse of a neovagina created with self-dilatation and treated with sacrospinous ligament suspension in a patient with MayerRokitansky-Küster-Hauser syndrome: a case report. Fertil Steril. 2010;93:267.e1-4.

[14] Fedele L, Frontino G, Motta F, Peruzzi E. Davydov's procedure for the treatment of neovaginal prolapse in Rokitansky syndrome. J Minim Invasive Gynecol. 2011;18:503-6.

[15] Christopoulos P, Cutner A, Vashisht A, Creighton SM. Laparoscopic sacrocolpopexy to treat prolapse of the neovagina created by vaginal dilation in Rokitansky syndrome. J Pediatr Adolesc Gynecol. 2011;24:e33-4.

[16] Toh VV, Bogne V, Bako A. Management of recurrent vault prolapse. Int Urogynecol J. 2012;23:29-34.

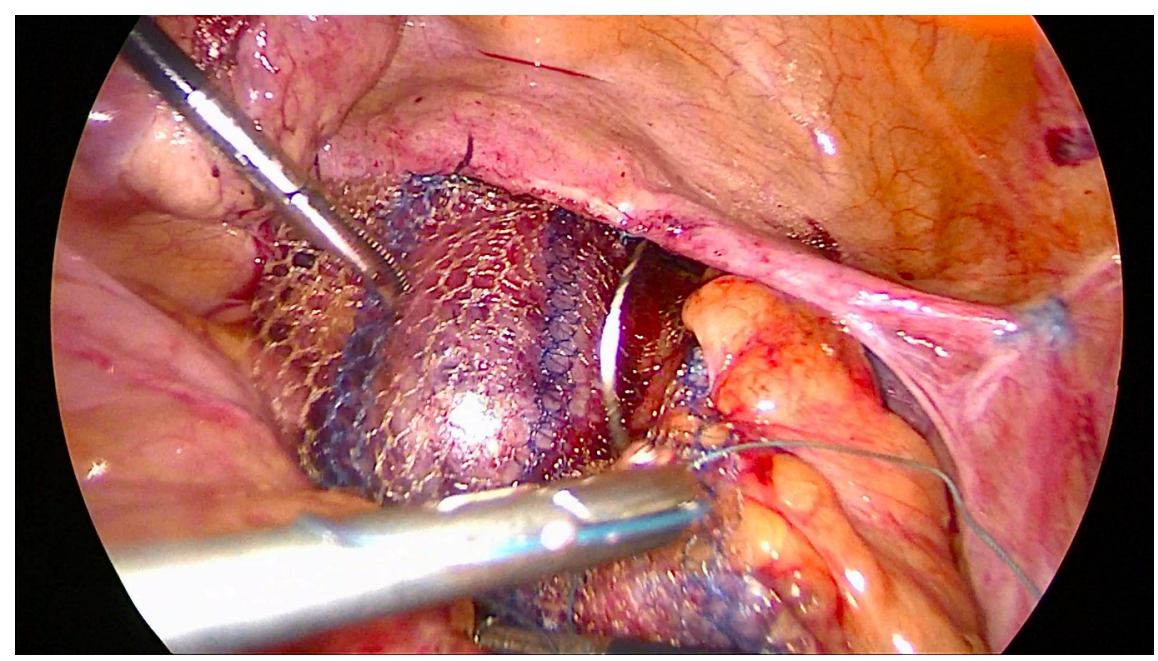



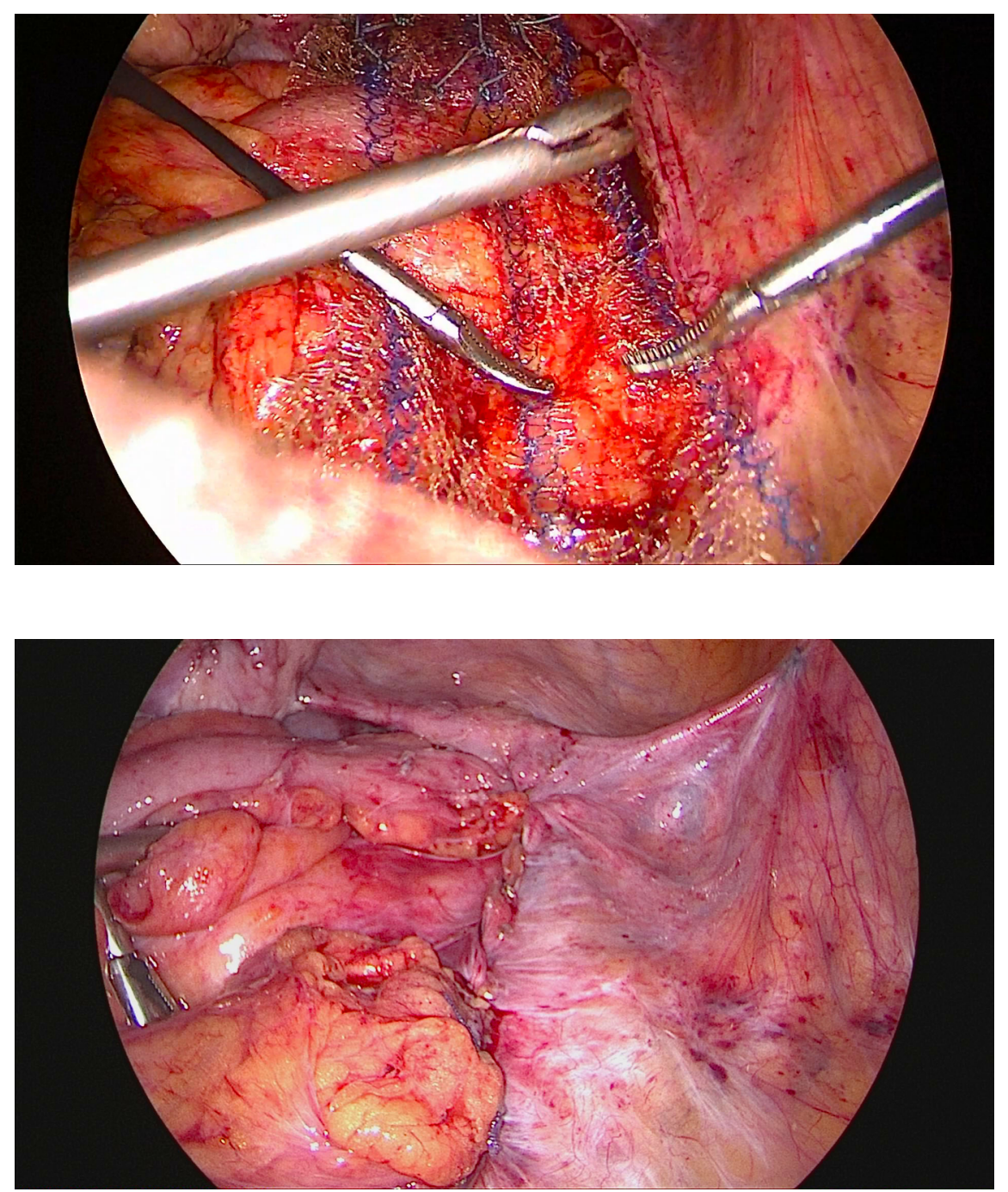


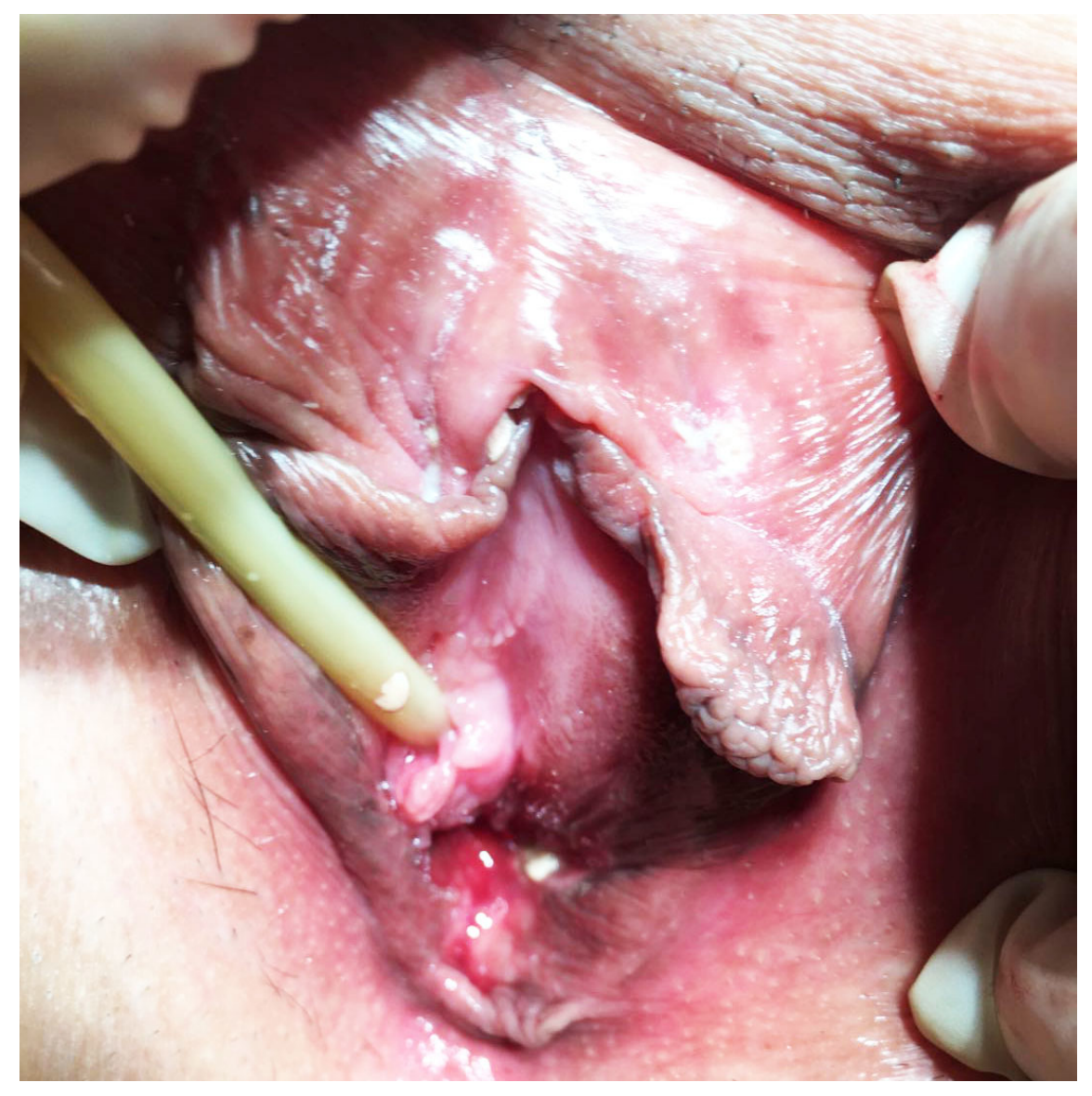

